\title{
A retrospective study of NENs and miR-224 promotes apoptosis of BON-1 cells by targeting PCSK9 inhibition
}

\author{
Jian'an Bai ${ }^{1}$, He Na ${ }^{2}$, Xiumei Hua ${ }^{2}$, Yaling Wei ${ }^{2}$, Tian $\mathrm{Ye}^{1}$, Yiqiang Zhang ${ }^{3}$, Guo Jian ${ }^{4}$, \\ Weiwen Zeng' ${ }^{2}$, Lijun Yan², Qiyun Tang ${ }^{2}$ \\ ${ }^{1}$ Department of Gastroenterology, Sir Run Run Hospital Affiliated with Nanjing Medical University, Nanjing, China \\ ${ }^{2}$ Department of Gastroenterology, The First Affiliated Hospital with Nanjing Medical University, Nanjing, China \\ ${ }^{3}$ Department of Radiology, Linyi People's Hospital, Dezhou, China \\ ${ }^{4}$ Department of Orthopaedics, Linyi People's Hospital, Dezhou, China \\ Correspondence to: Qiyun Tang, email: tay831@163.com
}

Keywords: NENs, epidemiology, LDL-C, PCSK9, miR-224

Received: February 29, $2016 \quad$ Accepted: December 16, $2016 \quad$ Published: December 28, 2016

\section{ABSTRACT}

Neuroendocrine neoplasms (NENs) represent relatively rare tumors. The lack of diagnostic, therapeutic method and prognostic factors makes them a challenge to us. We retrospectively reviewed the data of 205 NENs patients among which 157 cases were followed-up. Proprotein convertase subtilisin/kexin 9 (PCSK9), a regulator of low density lipoprotein cholesterol (LDL-C), was confirmed as a target gene of microRNA-224. We found an increased incidence of NENs from 2012 to 2015. Women were usually diagnosed at earlier stages than men $(P<0.05)$. Tumor grading was associated with primary tumor site, especially esophagus and cardia NENs all at G3 $(P<0.001)$. Age, tumor grading and LDL-C levels were independent risk factors of digestive NENs. Low LDL-C level was significantly correlated with survival rate and median overall survival (OS, $P<0.05)$. MicroRNA-224 agomir and PCSK9 siRNA could promote apoptosis and suppress proliferation, invasion of BON-1 cells $(P<0.05)$, but increase the level of glucocorticoid (GC, $P<0.05$ ). Taken together, age, tumor grading and LDL-C level are independent risk factors of NENs. The miR-224/PCSK9/GC axis binds to tumorigenesis and prognosis of pancreatic NENs (p-NENs).

\section{INTRODUCTION}

Neuroendocrine neoplasms (NENs), ever called carcinoid, represent a highly heterogeneous and intractable disease with multiple clinical manifestations [1]. In the latest 30 years, the incidence of this disease is obviously increased with a faster growing speed than other tumors [2]. The overall incidence of digestive NENs has increased by $720 \%$ from 1972 to 2004 [3]. Most NENs are bioactive nonfunctional tumors, which lead to an early diagnostic challenge.

The acknowledged primary tumor site, grade and metastasis have been demonstrated as prognostic factors of NENs which are related to the 5-year survival rate [4, 5]. However, there is almost no other prognostic factor. As a recently discovered regulator of low density lipoprotein cholesterol (LDL-C), proprotein convertase subtilisin/ kexin 9 (PCSK9) has been reported in early onset of heart disease family. It directly binds with LDL-C receptor
(LDLR) on stem cells, resulting in lysosomal degradation of LDLR complex in liver and following accumulation of LDL-C in serum $[6,7]$. Nevertheless, there is no report towards PCSK9/LDL-C and tumorigenesis.

MicroRNAs (miRNAs) are endogenously expressed non-coding RNAs which can bind to 3-untranslated region (UTR) of mRNAs [8]. Previous studies indicated the crucial role of miR-224 in solid tumors involving colorectal cancer, prostate cancer and hepatocellular carcinoma [9-11]. But there is no study on the effect of miR-224 in NENs.

The current paper retrospectively analyzed 205 cases with NENs and discovered LDL-C level as an independent risk factor of pancreatic NENs (p-NENs). Then, we decoded its mechanisms via miR-224/PCSK9/GC axis mediated cellular apoptosis, proliferation and invasion in p-NENs for the first time. Moreover, we demonstrated PCSK9 as a direct target of miR-224 and increased miR-224 or decreased PCSK9 could promote apoptosis and suppress proliferation, invasion of BON-1 cells in p-NENs. This may help us to 
evaluate the prognosis of $\mathrm{p}-\mathrm{NENs}$ patients and provide a new target to the treatment of p-NENs.

\section{RESULTS}

\section{Epidemiology}

A total of 205 NENs were involved and showed a significant increase in incidence of NENs from $2012(\mathrm{n}=$ $20)$ to $2015(\mathrm{n}=77$, Figure $1 \mathrm{~A})$. There were 89 patients (43.4\%) with NENs G1, 31 patients $(15.1 \%)$ with NENs G2, 80 patients $(39 \%)$ with NENs G3 and 5 patients $(2.4 \%)$ with mixed adenoneuroendocrine carcinoma (MANEC). Mean age of G3 and MANEC was older than

A

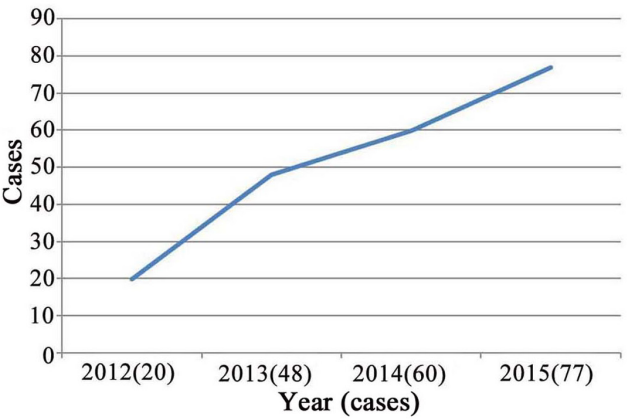

C

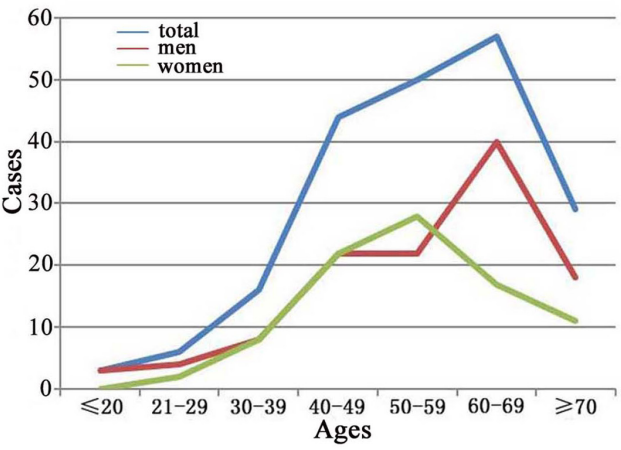

E

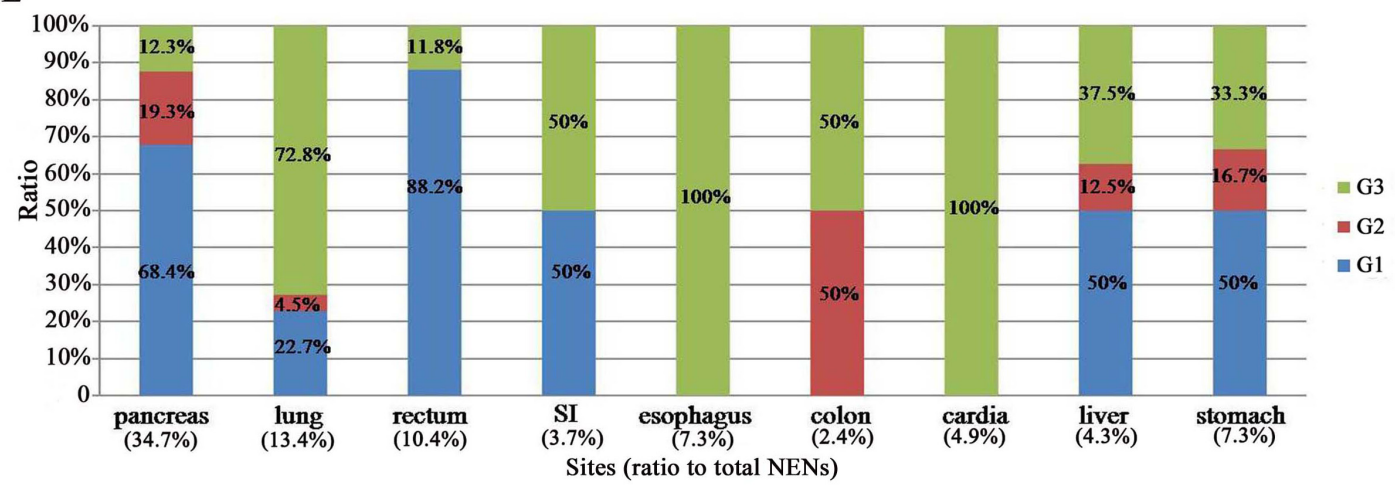

G1 and G2 with no significant difference $(P=0.213$, Figure 1B). The most common age of NENs was 60 to 69 years in men while 50 to 59 years old in women $(P<0.05$, Figure $1 \mathrm{C})$. Tumor grading was strongly associated with age, especially $>60$ years $(P<0.001$, Figure 1D).

The most common primary tumor site was pancreas ( $\mathrm{n}=57 ; 34.7 \%)$, followed by lung $(\mathrm{n}=22 ; 13.4 \%)$, rectum $(\mathrm{n}=17 ; 10.4 \%)$, esophagus $(\mathrm{n}=12 ; 7.3 \%)$, stomach $(\mathrm{n}$ $=12 ; 7.3 \%)$, cardia $(\mathrm{n}=8 ; 4.9 \%)$, small intestine $(\mathrm{SI}, \mathrm{n}$ $=6 ; 3.7 \%$ ). Tumor grading was strongly associated with primary tumor site, especially esophagus and cardia all at NENs G3. However, pancreatic and rectal NENs were always at $\mathrm{G} 1(P<0.001$, Figure 1E). Clinical characteristics of these patients were presented in Table 1. About $91.7 \%$

B

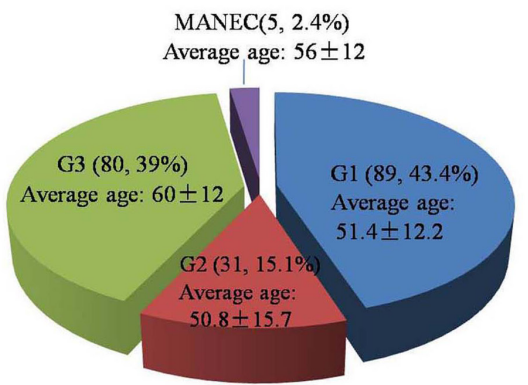

D

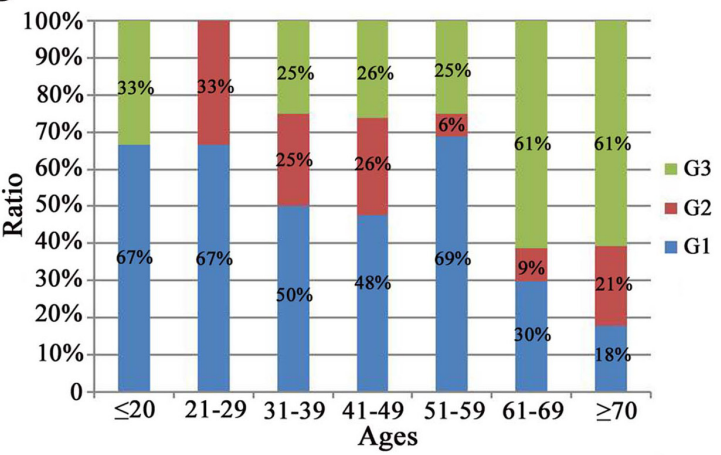

Figure 1: Epidemiology of 205 NENs patients. A. a significant increase was shown in the incidence of NENs from 2012 to 2015 B. distribution of patients according to stage and the mean age at diagnosis. C. the most common age of NENs in men and women. D. the relationship between tumor grading and the age. E. the common primary tumor site and the relationship between tumor grading and the primary tumor site. 
Table 1: Clinical characteristics of NENs patients

\begin{tabular}{lccccc}
\hline & abdominal pain & diarrhea & flush & syncope & hemoptysis \\
\hline pancreas & $16(22.2 \%)$ & 0 & $4(5.6 \%)$ & $11(15.5 \%)$ & 0 \\
cardia & $3(30 \%)$ & 0 & 0 & 0 & 0 \\
SI & $3(37.5 \%)$ & 0 & 0 & 0 & 0 \\
colon & $1(25 \%)$ & 0 & 0 & 0 & 0 \\
rectum & $4(19 \%)$ & $2(9.5 \%)$ & 0 & 0 & 0 \\
stomach & $1(6.7 \%)$ & 0 & 0 & 0 & 0 \\
liver & 0 & 0 & 0 & 0 & $11(40.7 \%)$ \\
lung & 0 & 0 & 0 & 0.0
\end{tabular}

NENs were nonfunctioning tumors. Abdominal pain was the most common complaint (only $13.7 \%$ of 205 cases).

\section{Levels of serum lipid}

The average levels of serum lipid in NENs patients were significantly lower than the healthy examinees including levels of total triglyceride (TG), total cholesterol (TC), high density lipoprotein cholesterol (HDL-C) and LDL-C (Supplementary Table 1), especially the LDL-C level $(2.55 \pm 0.8$ vs $3.5 \pm 0.85 \mathrm{mmol} / \mathrm{L}, P<0.001$, Supplementary Figure 1A).

There were more cases with high LDL-C $(>4.1$ $\mathrm{mmol} / \mathrm{L})$ in healthy examinees than those in NENs patients $(42 / 200$ vs $9 / 205, P<0.001)$ and more cases with low LDL-C $(\leq 2.6 \mathrm{mmol} / \mathrm{L})$ in NENs patients than those in healthy examinees $(26 / 200 v s 61 / 205, P=0.001$, Supplementary Table 2).

\section{Survival}

Among the 157 cases followed up, patients less than 60 years old were associated with better prognosis than over 60 years group $(P=0.018$, Figure 2A). Diagnosis of NENs G1 was associated with better prognosis than $\mathrm{G} 2$ and $\mathrm{G} 3(P=0.006$, Figure 2B). However, there was no significant correlation between survival and chemoradiotherapy $(P=0.93$, Figure 2C), level of LDL-C $(P=0.08$, Figure 2D), recurrence $(P=0.297$, Figure $2 \mathrm{E})$ and metastasis $(P=0.11$, Figure $2 \mathrm{~F})$.

Among the 116 cases of digestive NENs followed up, patients less than 60 years old were associated with better prognosis than over 60 years group $(P=0.03$, Figure 3A). Diagnosis of NENs G1 was associated with better prognosis than $\mathrm{G} 2$ and $\mathrm{G} 3(P=0.023$, Figure $3 B)$. Patients with high LDL-C level were associated with better prognosis than low LDL-C level group ( $P$ $=0.027$, Figure 3D). However, there was no significant correlation between survival and chemoradiotherapy ( $\mathrm{P}$ $=0.077$, Figure 3C), recurrence $(P=0.091$, Figure 3E) and metastasis $(P=0.06$, Figure $3 \mathrm{~F})$. Otherwise, patients with high LDL-C level owned 4.738-fold of survival rate than low LDL-C level group (95\% CI, 1.424 to $15.772, P$ $=0.019)$. LDL-C level was significantly correlated with survival rate $(\mathrm{r}=-0.23, P=0.013)$ but not significantly correlated with survival time $(\mathrm{r}=-0.055, P=0.557)$. In detail, the median overall survival (OS) in low LDL-C level group was $50.6 \pm 16.2$ months, $56.9 \pm 11.7$ months in normal LDL-C level group (2.6-4.1 mmol/L) and 51.1 \pm 7.6 months in high LDL-C level group $(P=0.039)$.

\section{PCSK9 is the direct target of miR-224}

PCSK9 was an effective regulator of LDL-C as previous reported. We detected expression of PCSK9 in tissue samples of p-NENs and found a significantly increase than para-tumor tissues ( $P<0.01$, Figure 4A). Therefore, we chose it as the following target. First, we used miRNA target predicting programs (TargetScan) to discover PCSK9 as a target of miR-224. Then, luciferase reporter was performed to confirm PCSK9 being targeted by miR-224. PCSK9 reporter was significantly decreased upon co-transfection with miR-224 agomir, but not in control group with mutated sequence $(P<0.05$, Figure $4 \mathrm{~B})$.

Upon transfection with miR-224 agomir, miR224 level was significantly increased $(P<0.01$, Figure 4C). Meanwhile, endogenous PCSK9 expression was significantly reduced at mRNA level $(P<0.05$, Figure 4D) and protein level $(P<0.05$, Figure 4E). Therefore, we believed that PCSK9 was a direct target of miR-224.

\section{miR-224 agomir or siPCSK9 promotes apoptosis and suppresses proliferation, invasion of $\mathrm{BON}-1$ cells in vitro}

Then, we detected cleaved caspase-3 level which was a "apoptotic executor" in mitochondrial apoptosis [12]. A significantly increased cleaved caspase-3 level was found in miR-224 agomir or siPCSK9 transfected 
BON-1 cells than negative control (NC) group $(P<0.05$, Figure 4E). The percentages of cell death or TUNELpositive cells were about $3 \%$ in miR-NC group and up to $8.9 \%$ in miR-224 agomir group ( $P<0.05$, Figure $5 \mathrm{~A})$. Meanwhile, it was $2.7 \%$ in siNC group and up to $8.2 \%$ in siPCSK 9 group $(P<0.05$, Figure 5A). Furthermore, we found a significantly lower proliferation potential in miR-224 agomir transfected BON-1 cells than miR-NC group after $72 \mathrm{~h}(P<0.05$, Figure 5B) with CCK 8 assay. The same result was seen in siNC and siPCSK9 group $(P$ $<0.05$, Supplementary Figure 1B).

B

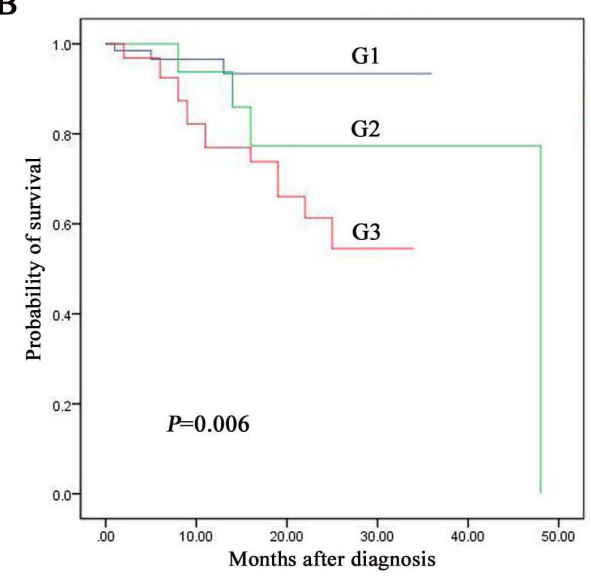

D

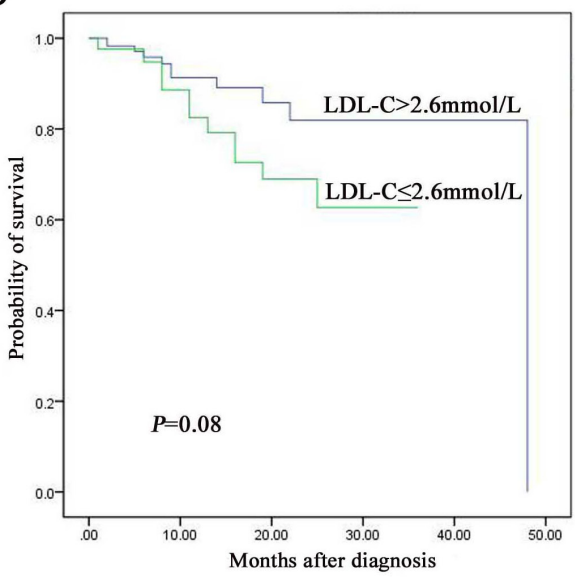

F

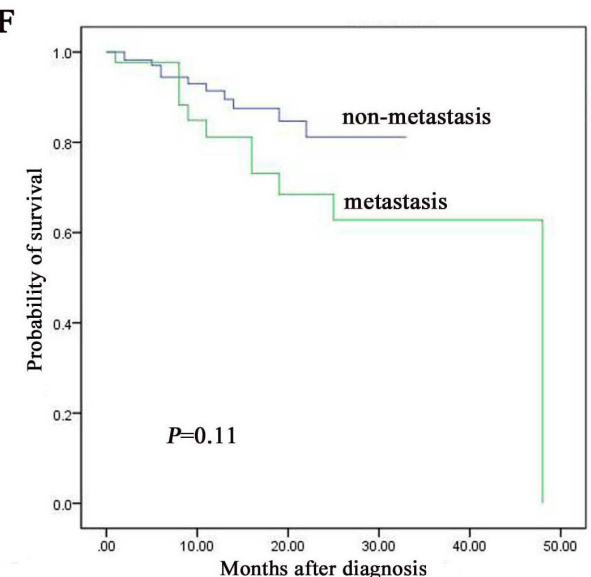

Figure 2: The prognostic factors assay by Kaplan-Meier analysis among the 157 NENs patients followed up. A. age was associated with prognosis of NENs. B. grading was associated with prognosis of NENs. C. chemoradiotherapy was not associated with prognosis of NENs. D. level of LDL-C was not associated with prognosis of NENs. E. recurrence was not associated with prognosis of NENs. F. metastasis was not associated with prognosis of NENs. 
A

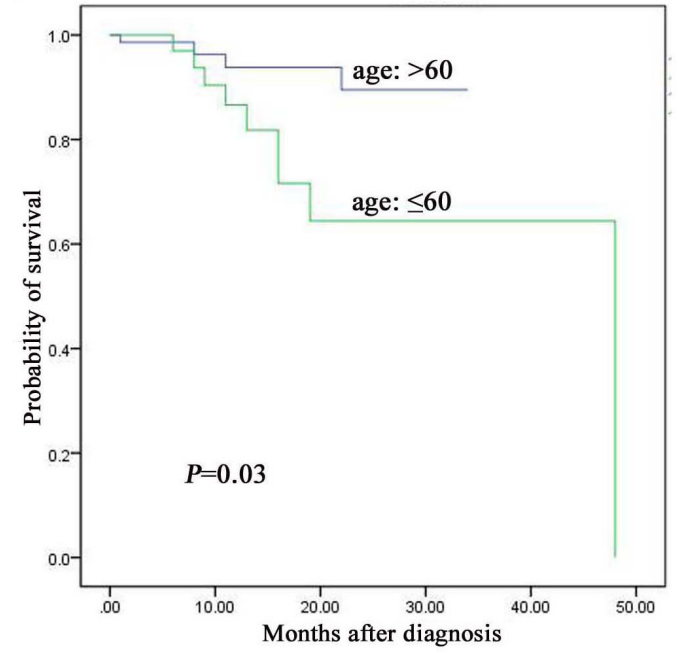

$\mathrm{C}$

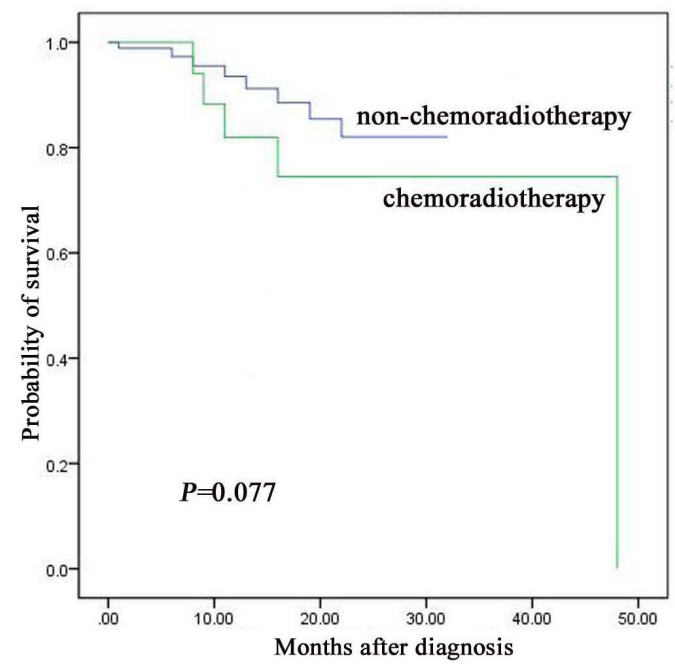

$\mathrm{E}$

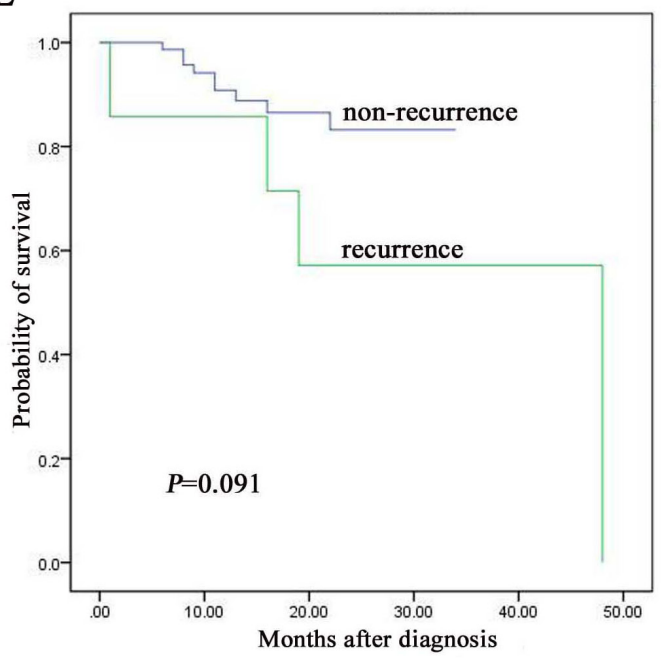

B

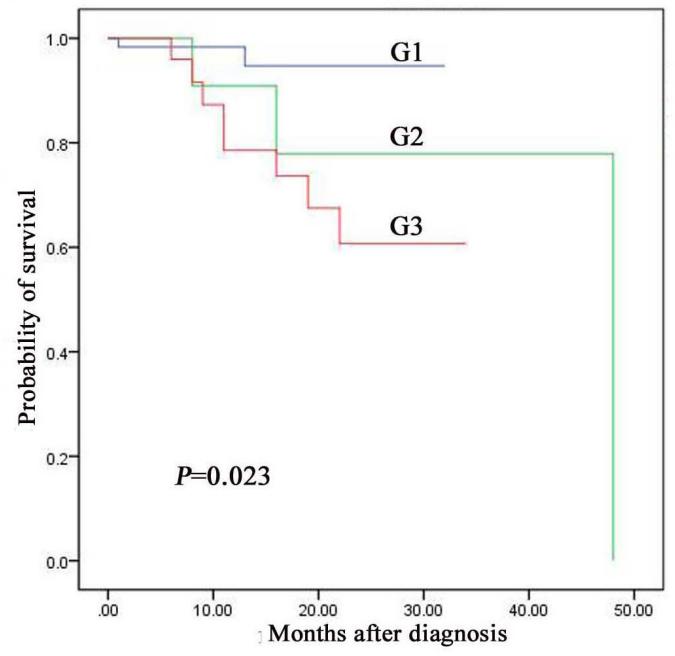

D

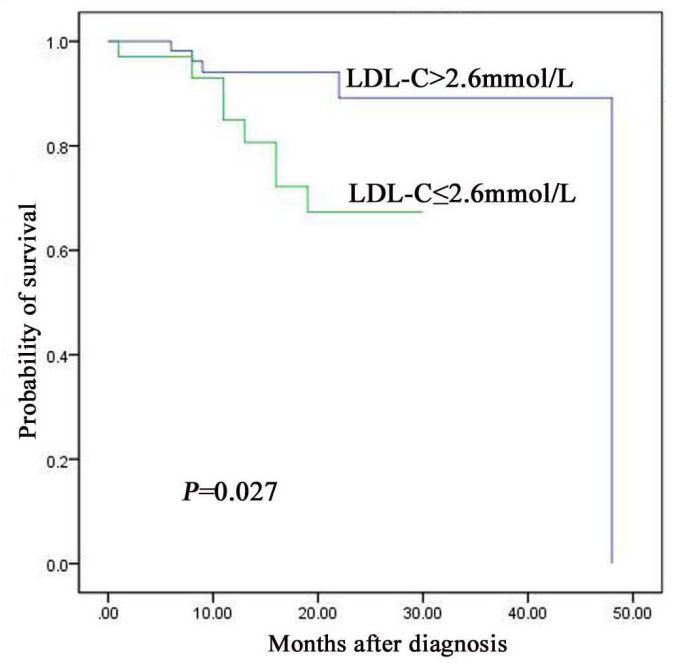

F

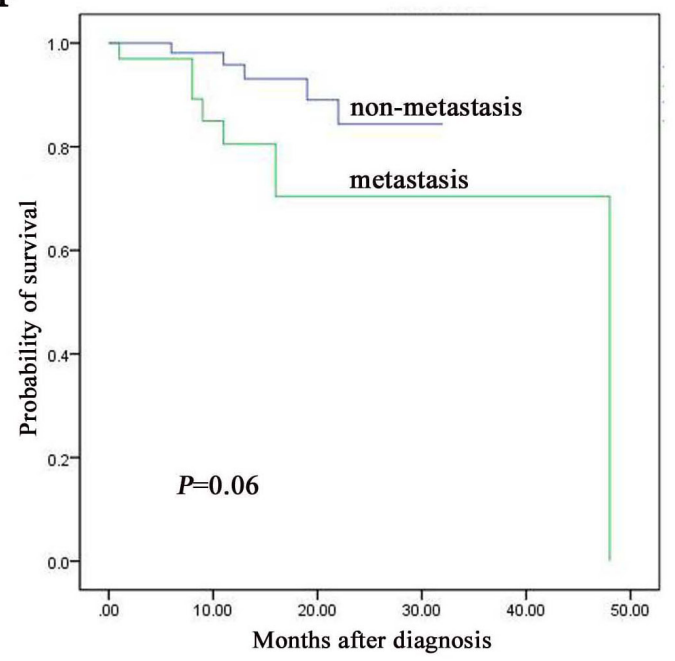

Figure 3: The prognostic factors assay by Kaplan-Meier analysis among the 116 digestive NENs patients followed up. A. age was associated with prognosis of digestive NENs. B. grading was associated with prognosis of digestive NENs. C. chemoradiotherapy was not associated with prognosis of digestive NENs. D. level of LDL-C was associated with prognosis of digestive NENs. E. recurrence was not associated with prognosis of digestive NENs. F. metastasis was not associated with prognosis of digestive NENs. 
A
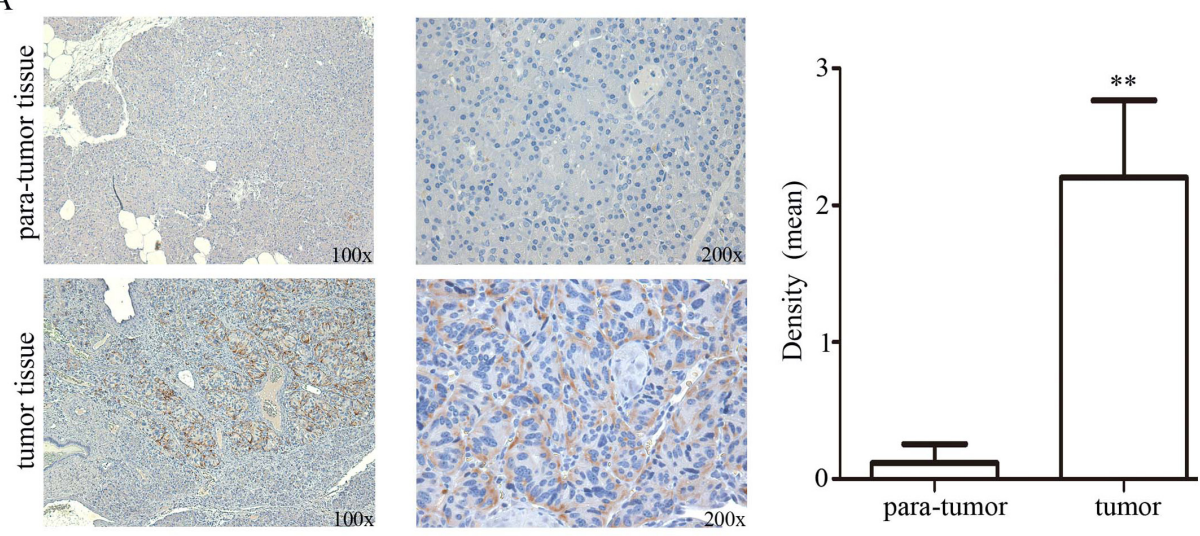

$\mathrm{B}$

PCSK9 3' UTR(1217) WT 5'...UUUUAUUAAUAUGGUGACUUU....'

miR-224 3'...UUGCCUUGGUGAUCACUGAAC... 5

PCSK9 3' UTR Mut 5'...UUUUAUUAAUAACCACUGAAU....'

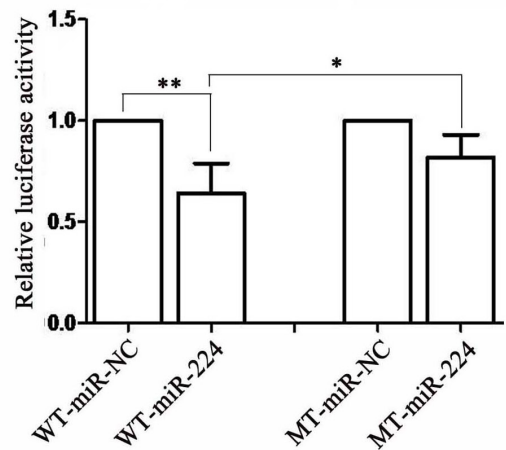

$\mathrm{C}$

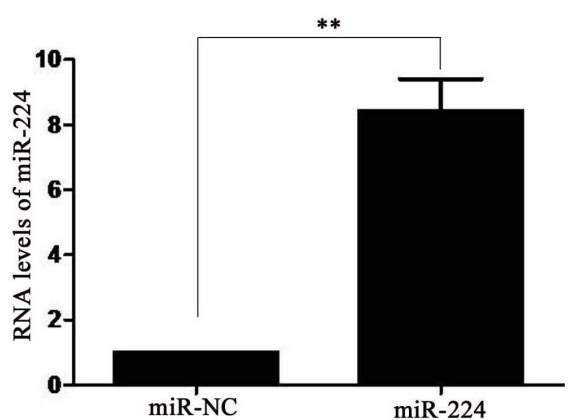

E

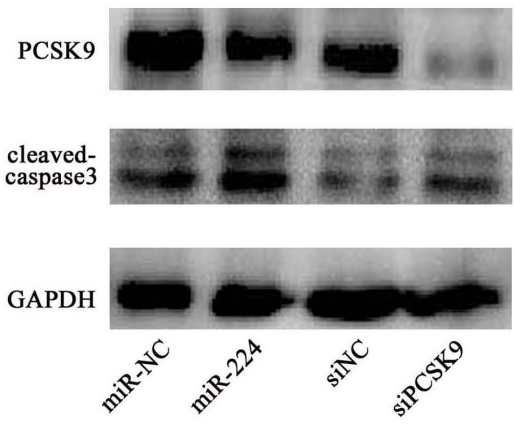

$\mathrm{D}$
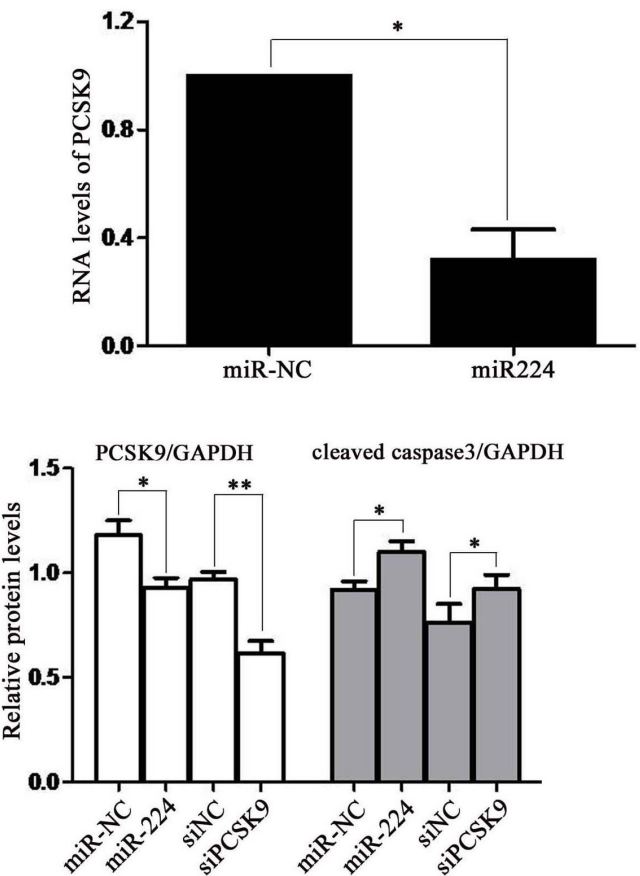

Figure 4: miR-224 down-regulates PCSK9 expression by directly targeting its 3'-UTR. A. PCSK9 expression was increased in tissue samples of p-NENs. Immunohistochemistry of PCSK9 in tumor and para-tumor samples from patients with NENs (n=6, 3 males and 3 females). There was almost non-staining of PCSK9 in the para-tumor samples, while strong staining of PCSK9 in tumor samples. B. RNA sequence alignment showed the 3'-UTR of PCSK9 mRNA contains a complementary site for miR-224. Dual luciferase reporter assay was performed to confirm the miR-224 binding target. C, D. RT-PCR analysis showed that the miR-224 expression was up-regulated and PCSK9 expression was down-regulated in BON-1 cells upon miR-224 transfection at mRNA levels. E. western blot analysis showed that the endogenous PCSK9 expression was significantly reduced at protein level followed by increased expression of cleaved caspase-3 upon miR-224 agomir or siPCSK9 transfection. 
A
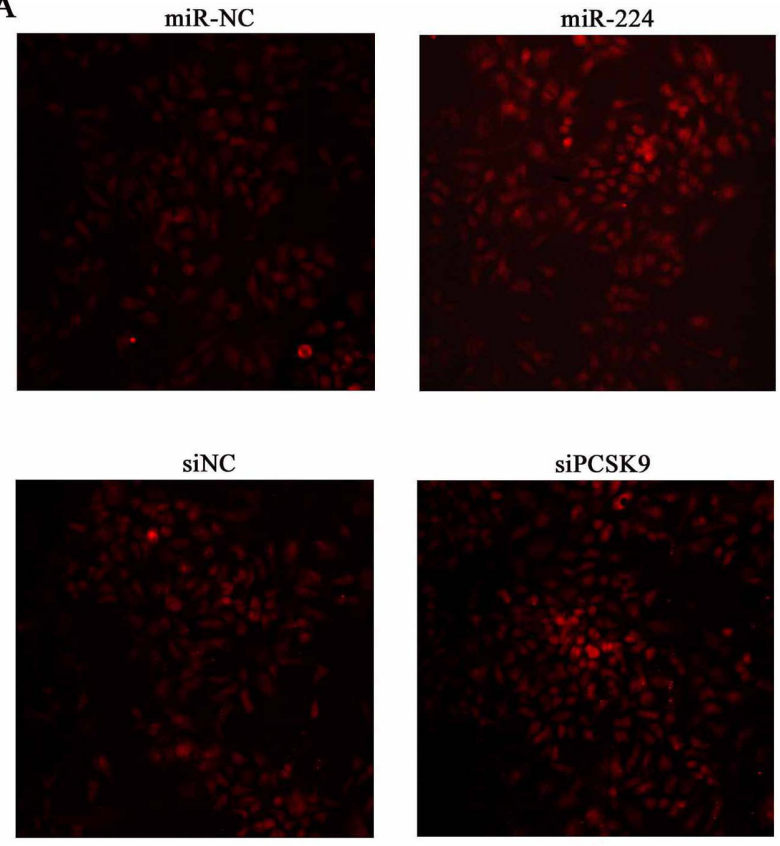

B

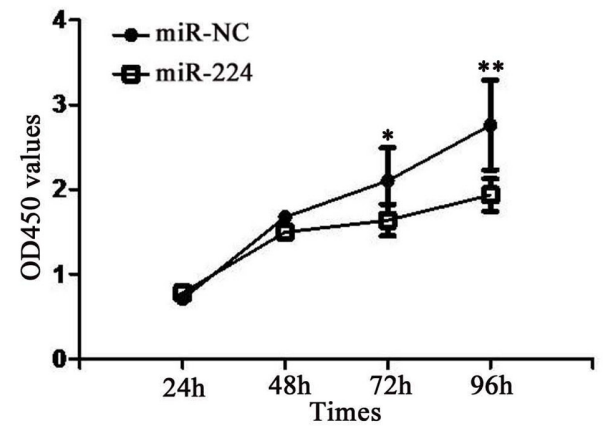

C

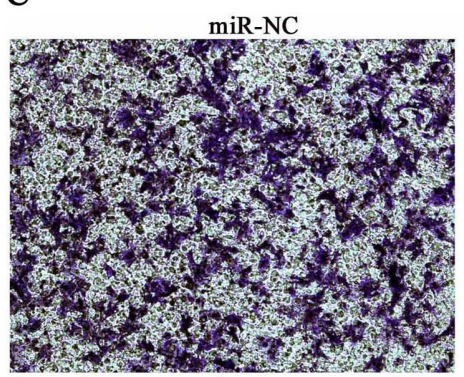

$\operatorname{siNC}$

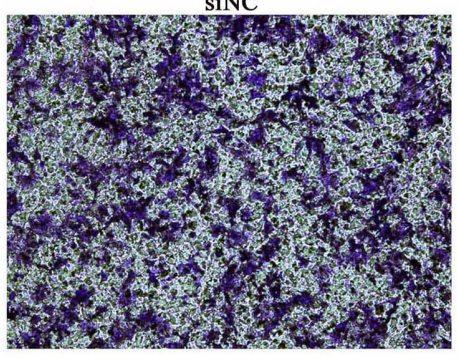

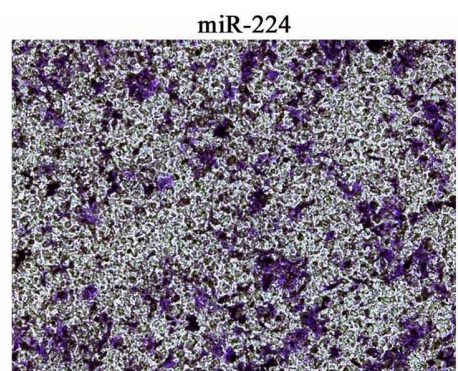

siPCSK9

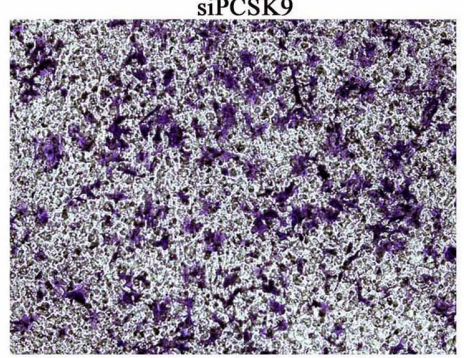

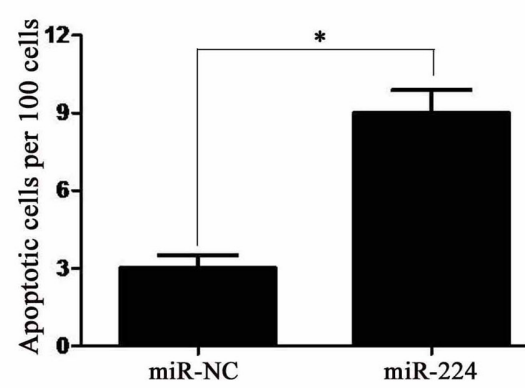

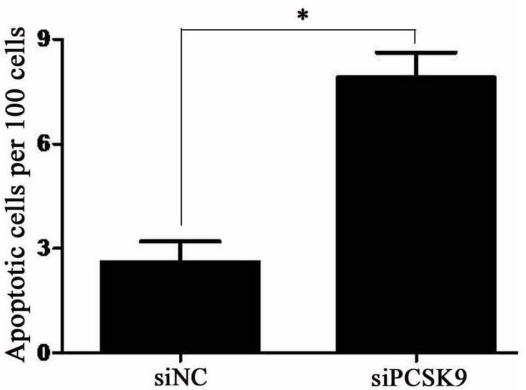

D

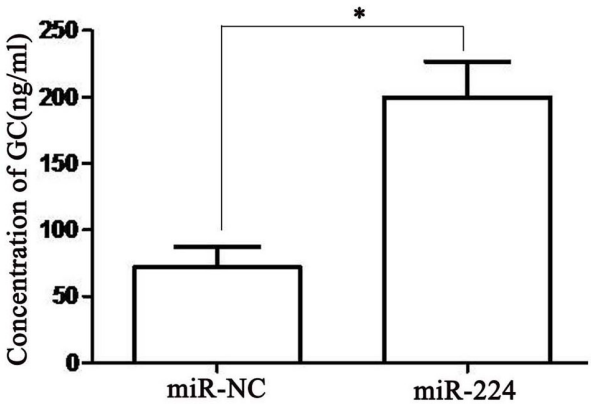

$\operatorname{miR}-224$

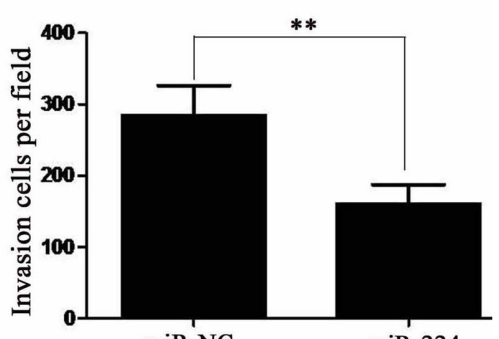

miR-NC

$\operatorname{miR}-224$

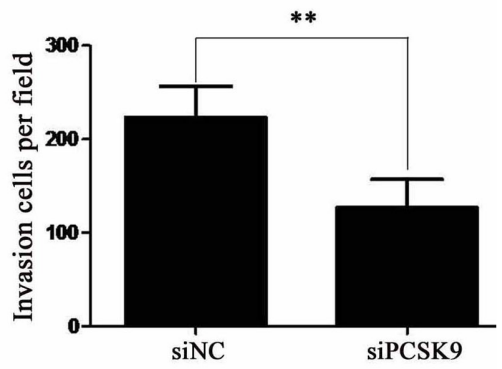

Figure 5: miR-224 agomir or siPCSK9 promotes apoptosis and suppresses proliferation, invasion of BON-1 cells. A. the TUNEL-positive cells was about $3 \%$ in miR-NC group and up to $8.9 \%$ in miR-224 agomir group. Meanwhile, it was $2.7 \%$ in siNC group and up to $8.2 \%$ in siPCSK9 group. B. CCK8 assay showed significantly lower proliferation potential in miR-224 agomir transfected cells than control group after $72 \mathrm{~h}$. C. transwell assay showed significantly less invasive cells in miR-224 agomir group or siPCSK9 group than in control group. D. ELISA assay showed an increased level of GC in miR-224 agomir group than in miR-NC group. 
In transwell assay, there were average of 282 invasive cells in miR-NC group and only 138 invasive cells in miR-224 agomir group. Meanwhile, there were average of 215 invasive cells in siNC group and only 127 invasive cells in siPCSK 9 group $(P<0.05$, Figure 5C).

Therefore, we performed an enzyme-linked immuno sorbent assay (ELISA) assay to measure GC level and found an increased level of GC in miR-224 agomir group than in miR-NC group $(P<0.05$, Figure 5D) and the same result in siPCSK9 group than siNC group $(P<0.05$, Supplementary Figure 1C).

\section{No tumor formation in the nude-mice with $\mathrm{BON}$ - 1 cells}

To finally confirm the function of miR-224 and PCSK9, we performed tumor formation assay in vivo. About $5 \times 10^{6} \mathrm{BON}-1$ cells/mouse and $1 \times 10^{7} \mathrm{BON}-1$ cells/ mouse with or without pre-transfection were employed. Regrettably, there was no tumor formation in any mice. These results might indicate disability of BON-1 cells in tumor formation.

\section{DISCUSSION}

Since 1907 , we have paid attention to them for a long way of more than 100 years. However, that is still a big challenge about early diagnosis and effective treatment $[3,13]$. Here, we retrospectively analyzed 205 cases of NENs in our hospital about clinical data and demonstrated a significant increase in incidence of NENs in recent 4 years.

Some discrepancies to previous studies or results still exist: (1) the most common primary tumor site is pancreas, followed with small intestine; (2) tumor grading was strongly associated with primary tumor site, especially esophagus and cardia all at NENs G3; (3) level of LDL-C is significantly correlated with the survival rate and OS [2-4, 14-16]. The most important thing is that esophageal and cardiac NENs need more attentions owing to the bad differentiation.

LDL-C is known as adverse cholesterol and raw material of GC synthesis which plays a critical role in cellular apoptosis $[17,18]$. Several studies have showed effect of GC on lipid metabolism [19], but seldom explored effect of lipid level on GC synthesis. Ronald et al have reported for the first time an inhibitory role of LDLR in GC production [20]. However, we found a discrepant result in our paper. An increasing level of GC was shown upon up-regulation of miR-224 or down-regulation of PCSK9. We considered it as a dynamic state. In detail, LDLR-mediated degradation of LDL-C released more raw material of GC synthesis, inducing accumulation of GC in plasma.

PCSK9 is responsible for degradation of the LDL-C receptor at the cell surface, and thus has a direct effect on intracellular and serum LDL-C levels [21]. It has only been reported as a target gene of two miRNA (miR-27a and miR-124) [22, 23]. However, relationship between miR-224 and PCSK9 is still unknown. MiR-224 has been reported to correlated with apoptosis, proliferation, cell cycle and invasion of several cancers especially the lung cancer and colon cancer [24-26]. In our study, we demonstrated PCSK9 as a direct target of miR-224. Moreover, we uncovered that increased miR-224 or decreased PCSK9 could promote apoptosis and suppress proliferation, invasion of BON-1 cells in pNENs.

In conclusion, we demonstrate that age, tumor grading and LDL-C level are independent risk factors of digestive NENs. Level of LDL-C is significantly correlated with survival rate and OS of NENs patients. MiR-224/ PCSK9/GC axis closely correlated with carcinogenisis and prognosis of $\mathrm{p}$-NENs which may provide a new target to treatment of p-NENs.

\section{MATERIALS AND METHODS}

\section{Patients and immunohistochemistry}

Epidemiological study involved 205 patients (117 men, 88 women) from database of record room in our hospital from 2012 to 2015, with diagnosis of NENs according to WHO 2010 criteria [2]. A total of 157 patients including 116 cases of digestive NENs were successfully followed-up. We summarized clinical features and prognostic factors including age, sex, grading (G1, G2, G3 without MANEC), distant metastases at diagnosis, chemoradiotherapy and relapse. Date for survival analyses was from diagnosis until death. Serum lipid levels of NENs patients and 200 healthy examinees with similar age and sex were retrospectively collected at the first coming to our hospital before surgery. This study was approved by ethics committee of the First Affiliated Hospital with Nanjing Medical University.

Tissues of p-NENs patients $(n=6)$ were gained from pathology department in our hospital. Immunohistochemistry assay was performed according to manufacturer's protocol. The immunohistochemical kit was used (Beyotime Co., Ltd, China) for all procedures. Para-tumor samples were served as negative controls. These slices were visualized with a microscope (IX71; Olympus Corp., Tokyo, Japan).

\section{Cell culture and transfection}

Human p-NENs cell lines BON-1 was obtained from American Type Culture Collection and authenticated with short tandem repeat (STR, JiaPeng Co., Ltd, China). Cells were cultured in DMEM medium (Hyclone) added with $10 \%$ FBS (Siencell, USA) conventionally.

The miRNA-224 agomir, agomir negative control, PCSK9 siRNA (siPCSK9) and siRNA negative 
control (siNC) were designed (GenePharma Co.,Ltd, China). The sequence of miRNA-224 agomir was 5'-CAAGUCACUAGUGGUUCCGUU-3' (sense), 5'-CGGA ACCACUAGUGACUUGUU-3' (antisense). Sequence of siRNA against PCSK9 was shown as follows: 5'-CCAAGAUCCUGCAUGUCUUTT-3' (sense), 5'-AAG ACAUGCAGGAUCUUGGTT-3' (antisense). Sequence of siRNA negative control was as follows: 5'-UUCU CCGAACGUGUCACGUTT-3' (sense), 5'- ACGU GACACGUUCGGAGAATT-3' (antisense). Transfected experiments were performed with Lipofectamine 2000 (Invitrogen) according to manufacturer's procedure. BON1 cells were respectively cultured with miRNA agomir, siRNA, or negative control for 48 hours.

\section{MiRNA target prediction and luciferase reporter assay}

Potential targeted relation of PCSK9 and miR-224 was predicted with Target-Scan. Results were assessed with luciferase reporter assay in BON-1 cells. BON-1 cells were co-cultured with miR-224 agomir or negative control $(50 \mathrm{nmol} / \mathrm{L})$, and then wild-type (WT) or mutant (MT) reporter plasmid $(0.5 \mathrm{mg})$. Cells were collected 48 hours after transfection and measured with dual-luciferase reporter assay system (Promega, USA). Data were normalized to renilla luciferase signal.

\section{Western blot}

For immunoblot analysis, about $80 \mu \mathrm{g}$ of routinely distracted cellular protein was resolved on a $10 \%$ sodium dodecyl sulfate polyacrylamide electrophoresis gel. PCSK9 (1:6000), cleaved-caspase 3 (1:6000) and glyceraldehyde-3-phosphate dehydrogenase (GAPDH, 1:6000) were served as primary antibodies (Abcam, USA). GAPDH was served as an internal control. The membranes were exposed to bands with a Chemiluminescent HRP Substrate (Millipore, MA, USA).

\section{RNA isolation and real-time PCR}

Trizol RNA extraction kit, reverse transcription kit and SYBR Green PCR Kit (Takara, Japan) were used for real-timePCR assay according to the manufacture's protocol on StepOne-PCR System (Applied Biosystems). Relative expression ratio of target genes was calculated by $2^{-\Delta \Delta \mathrm{CT}}$ method. Expression levels of miR-224 or PCSK9 were normalized to U6 or GAPDH, respectively. Specific primers of PCSK9 were 5'-AGACCCACCTCTCGCAGTC-3' (sense); 5'-GGAGTCCTCCTCGATGTAGTC-3' (antisense);

Primers of GAPDH were 5'-CTGGGCTACA CTGAGCACC-3' (sense); 5'-AAGTGGTCGTTGAGG GCAATG-3' (antisense). MiR-224 and U6 were designed and synthesized (Ribobio Co., Ltd, China).

\section{TUNEL and CCK-8 assay}

TdT-UTP nick-end labeling (TUNEL; Beyotime Co., Ltd, China) assay was performed according to manufacture's protocol. Fluorescein isothiocyanate (FITC)-labeled TUNEL-positive cells were imaged with a microscope. Cells with deep red fluorescence were defined as apoptotic cells.

A Cell Counting Kit-8 (CCK-8) assay was performed following manufacturer's protocol to evaluate cellular proliferation. Each sample was supplemented with $10 \mu \mathrm{l}$ tetrazolium substrate after transfection for $24,48,72$ and $96 \mathrm{~h}$. Optical density (OD) was then read at $450 \mathrm{~nm}$ in a spectrophotometer (Bio-Tek, USA).

\section{ELISA assay}

To evaluate levels of glucocorticoid, human Enzyme-linked immunosorbent assay (ELISA, Senbeijia Co., Ltd, China) was performed according to manufacturer's protocol. Absorbance was measured at $450 \mathrm{~nm}$ and levels of glucocorticoid were read out from respective standard curve.

\section{Transwell assay}

Cell invasion viability was performed by Transwell assay according to the protocol in previous studies [27]. Migrated cells were photographed and counted at 6 random fields of each group.

\section{Animals and tumor formation assays}

Animal experiments were supported by the Institute for Laboratory Animal Research at Nanjing Medical University. Twelve BALB/c nude mice (4 to 5 weeks old, females) were purchased from Animal Center of Jiangsu province. Pre-transfected BON-1 cells with miR-224 agomir or control agomir were collected in PBS. The highest concentration of $1 \times 10^{7}$ cells were subcutaneously injected into oxter of each mouse (4/ group). Situation and tumor formation were observed and recorded every 2 days.

\section{Statistical analysis}

Pictures drawing and statistical analysis were performed with GraphPad prism 5 and SPSS (version13.0). Differences between two groups were estimated with Student's $t$ test. Continuous variables were expressed as mean $\pm \mathrm{SD}$. The Pearson $\chi^{2}$ test or Fisher exact test was performed for crosstables. The univariate analysis was performed with Kaplan-Meier method and compared with log-rank tests for the survival rate. Pearson relevant analysis was calculated between levels of LDL-C and overall survival and OS. A $P$ value of less than 0.05 was considered statistically significant. 


\section{CONFLICTS OF INTEREST}

No conflicts of interest.

\section{FINANCIAL SUPPORT}

Sponsored by the National Natural Science Foundation of China, No. 81470806; the National Natural Science Foundation of Jiangsu Province, No. BK20141496; the Public Health Ministry of Jiangsu Province in the Talents in Medical Science Program. No. RC201179.

\section{Author contributions}

Jian'an Bai and $\mathrm{He} \mathrm{Na}$ contributed equally to this study; Jian'an Bai and Qiyun Tang concept and design the experiment; Jian'an Bai, Yaling Wei, He Na, Xiumei Hua, Tian Ye, Lijun Yan, and Weiwen Zeng develop the methodology; Jian'an Bai, Yaling Wei, He Na, Yiqiang Zhang and Guo Jian acquisited the data; Jian'an Bai, Tian Ye and Qiyun Tang analyzed and interpretated of data; Jian'an Bai written the manuscript; Qiyun Tang reviewed and revised the manuscript; Jian'an Bai, Tian Ye, Yiqiang Zhang, Guo Jian and Qiyun Tang supported technology and materials; Qiyun Tang supervised this study.

\section{REFERENCES}

1. Klimstra DS, Modlin IR, Coppola D, Lloyd RV, Suster S. The pathologic classification of neuroendocrine tumors: a review of nomenclature, grading, and staging systems. Pancreas. 2010; 39:707-712.

2. Turaga KK, Kvols LK. Recent progress in the understanding, diagnosis, and treatment of gastroenteropancreatic neuroendocrine tumors. CA Cancer J Clin. 2011; 61:113-132.

3. Yao JC, Hassan M, Phan A, Dagohoy C, Leary C, Mares JE, Abdalla EK, Fleming JB, Vauthey JN, Rashid A, Evans DB. One hundred years after "carcinoid": epidemiology of and prognostic factors for neuroendocrine tumors in 35,825 cases in the United States. Journal of clinical oncology. 2008; 26:3063-3072.

4. Lewkowicz E, Trofimiuk-Muldner M, Wysocka K, Pach D, Kieltyka A, Stefanska A, Sowa-Staszczak A, Tomaszewska R, Hubalewska-Dydejczyk A. Gastroenteropancreatic neuroendocrine neoplasms: a 10-year experience of a single center. Polskie Archiwum Medycyny Wewnetrznej. 2015; 125:337-346.

5. Cives M, Strosberg J. An update on gastroenteropancreatic neuroendocrine tumors. Oncology. 2014; 28:749-756, 758.

6. Abifadel M, Varret M, Rabes JP, Allard D, Ouguerram K, Devillers M, Cruaud C, Benjannet S, Wickham L, Erlich D, Derre A, Villeger L, Farnier M, Beucler I, Bruckert E, Chambaz J, et al. Mutations in PCSK9 cause autosomal dominant hypercholesterolemia. Nature genetics. 2003; 34:154-156.

7. Shimada YJ, Cannon CP. PCSK9 (Proprotein convertase subtilisin/kexin type 9) inhibitors: past, present, and the future. European heart journal. 2015.

8. Lagos-Quintana M, Rauhut R, Lendeckel W, Tuschl T. Identification of novel genes coding for small expressed RNAs. Science. 2001; 294:853-858.

9. Ling H, Pickard K, Ivan C, Isella C, Ikuo M, Mitter R, Spizzo R, Bullock MD, Braicu C, Pileczki V, Vincent K, Pichler M, Stiegelbauer V, Hoefler G, Almeida MI, Hsiao A, et al. The clinical and biological significance of MIR-224 expression in colorectal cancer metastasis. Gut. 2015.

10. $\mathrm{Fu} \mathrm{H}$, He HC, Han ZD, Wan YP, Luo HW, Huang YQ, Cai C, Liang YX, Dai QS, Jiang FN, Zhong WD. MicroRNA-224 and its target CAMKK2 synergistically influence tumor progression and patient prognosis in prostate cancer. Tumour biology. 2015; 36:1983-1991.

11. Lan SH, Wu SY, Zuchini R, Lin XZ, Su IJ, Tsai TF, Lin YJ, Wu CT, Liu HS. Autophagy suppresses tumorigenesis of hepatitis B virus-associated hepatocellular carcinoma through degradation of microRNA-224. Hepatology. 2014; 59:505-517.

12. Green DR, Llambi F. Cell Death Signaling. Cold Spring Harbor perspectives in biology. 2015; 7.

13. Kunz PL. Carcinoid and neuroendocrine tumors: building on success. Journal of clinical oncology. 2015; $33: 1855-1863$

14. O'Toole D, Salazar R, Falconi M, Kaltsas G, Couvelard A, de Herder WW, Hyrdel R, Nikou G, Krenning E, Vullierme MP, Caplin M, Jensen R, Eriksson B, Frascati Consensus C and European Neuroendocrine Tumor S. Rare functioning pancreatic endocrine tumors. Neuroendocrinology. 2006; 84:189-195.

15. Garcia-Carbonero R, Sorbye H, Baudin E, Raymond E, Wiedenmann B, Niederle B, Sedlackova E, Toumpanakis C, Anlauf M, Cwikla J, Caplin M, O'Toole D, Perren A and all other Vienna Consensus Conference p. Consensus Guidelines for High Grade Gastro-Entero-Pancreatic (GEP) Neuroendocrine Tumours and Neuroendocrine Carcinomas (NEC). Neuroendocrinology. 2016.

16. Ploeckinger U, Kloeppel G, Wiedenmann B, Lohmann $\mathrm{R}$ and representatives of 21 German NETC. The German NET-registry: an audit on the diagnosis and therapy of neuroendocrine tumors. Neuroendocrinology. 2009; 90:349-363.

17. Yang N, Caratti G, Ince LM, Poolman TM, Trebble PJ, Holt CM, Ray DW, Matthews LC. Serum cholesterol selectively regulates glucocorticoid sensitivity through activation of JNK. The Journal of endocrinology. 2014; 223:155-166.

18. Lee TH, Nam JG, Lee HM, Kim BY, Kang MK, Bae WY, Hur DY, Park SK. Dexamethasone Induces Apoptosis of Nasal Polyp-Derived Tissue Cultures Through JNK 
and p38 MAPK Activation. Clinical and experimental otorhinolaryngology. 2014; 7:112-118.

19. van den Beukel JC, Boon MR, Steenbergen J, Rensen PC, Meijer OC, Themmen AP, Grefhorst A. Cold Exposure Partially Corrects Disturbances in Lipid Metabolism in a Male Mouse Model of Glucocorticoid Excess. Endocrinology. 2015; 156:4115-4128.

20. van der Sluis RJ, Van Eck M, Hoekstra M. Adrenocortical LDL receptor function negatively influences glucocorticoid output. The Journal of endocrinology. 2015; 226:145-154.

21. Tice JA, Kazi DS, Pearson SD. Proprotein Convertase Subtilisin/Kexin Type 9 (PCSK9) Inhibitors for Treatment of High Cholesterol Levels: Effectiveness and Value. JAMA internal medicine. 2016; 176:107-108.

22. Alvarez ML, Khosroheidari M, Eddy E, Done SC. MicroRNA-27a decreases the level and efficiency of the LDL receptor and contributes to the dysregulation of cholesterol homeostasis. Atherosclerosis. 2015; 242:595-604.

23. Lee HS, Seok H, Lee DH, Ham J, Lee W, Youm EM, Yoo JS, Lee YS, Jang ES, Chi SW. Abasic pivot substitution harnesses target specificity of RNA interference. Nature communications. 2015; 6:10154.
24. Cui R, Kim T, Fassan M, Meng W, Sun HL, Jeon YJ, Vicentini C, Tili E, Peng Y, Scarpa A, Liang G, Zhang YK, Chakravarti A, Croce CM. MicroRNA-224 is implicated in lung cancer pathogenesis through targeting caspase-3 and caspase-7. Oncotarget. 2015; 6:21802-21815. doi: 10.18632/oncotarget.5224.

25. An F, Olaru AV, Mezey E, Xie Q, Li L, Piontek KB, Selaru FM. MicroRNA-224 Induces G1/S Checkpoint Release in Liver Cancer. Journal of clinical medicine. 2015; 4:1713-1728.

26. Cui R, Meng W, Sun HL, Kim T, Ye Z, Fassan M, Jeon YJ, Li B, Vicentini C, Peng Y, Lee TJ, Luo Z, Liu L, Xu D, Tili E, Jin V, et al. MicroRNA-224 promotes tumor progression in nonsmall cell lung cancer. Proceedings of the National Academy of Sciences of the United States of America. 2015; 112:E4288-4297.

27. Zhang Z, Wang J, Ji D, Wang C, Liu R, Wu Z, Liu L, Zhu D, Chang J, Geng R, Xiong L, Fang Q, Li J. Functional genetic approach identifies MET, HER3, IGF1R, INSR pathways as determinants of lapatinib unresponsiveness in HER2-positive gastric cancer. Clinical cancer research. 2014; 20:4559-4573. 